\title{
Interleukin-17 promotes the development of cisplatin resistance in colorectal cancer
}

\author{
GUOLONG SUI ${ }^{1}$, YINGNA QIU ${ }^{2}$, HAIJUAN YU ${ }^{3}$, QINGBIN KONG ${ }^{1}$ and BAOWEN ZHEN ${ }^{4}$ \\ Departments of ${ }^{1}$ Gastrointestinal Surgery and ${ }^{2}$ Anesthesia, Weihai Central Hospital, Weihai, Shandong 26440; \\ Departments of ${ }^{3}$ Nursing and ${ }^{4}$ Surgery, Naiman County People's Hospital, Tongliao, Inner Mongolia 028300, P.R. China
}

Received October 18, 2017; Accepted April 4, 2018

DOI: $10.3892 / \mathrm{ol} .2018 .9645$

\begin{abstract}
Cisplatin (DDP)-based anticancer therapy is an important chemotherapeutic strategy for the treatment of colorectal cancer. However, its beneficial effect is largely compromised by adverse reactions, and more importantly, by the development of drug resistance. Therefore, it is crucial to determine the potential mechanism underlying the development of DDP resistance in colorectal cancer. Interleukin-17 (IL-17) is a proinflammatory cytokine that has been found to serve an important role in the host defense during cancer development. It has been suggested that IL-17 is key to promoting the development of resistance to DDP in several major types of cancer. However, the role of IL-17 in DDP resistance in colorectal cancer has not been extensively investigated. In the present study, it was observed that IL-17 was significantly upregulated in colorectal tumor samples, compared with the adjacent tissues. Furthermore, IL-17 was found to promote the viability of HCT116 colorectal cells treated with DDP, whilst blocking IL-17 signaling leading to HCT116 cell apoptosis. IL-17 was also shown to regulate the expression of several apoptosis-related proteins, including phosphorylated-protein kinase B (p-Akt), apoptosis regulator BAX (Bax), apoptosis regulator $\mathrm{Bcl}-2(\mathrm{Bcl}-2)$ and serine/threonine-protein kinase mTOR (mTOR). These findings indicated that IL-17 facilitates the development of DDP resistance in colorectal cancer by inhibiting cancer cell apoptosis through targeting p-Akt, Bax, Bcl-2 and mTOR. Overall, the findings of the present study suggest that a combination of DDP and an IL-17 inhibitor may prove to be a highly efficient strategy for colorectal cancer treatment.
\end{abstract}

Correspondence to: Dr Baowen Zhen, Department of Surgery, Naiman County People's Hospital, 2762 Naiman County Street, Daqintala, Tongliao, Inner Mongolia 028300, P.R. China

E-mail: baowenzhennmg@outlook.com

Key words: interleukin-17, cisplatin, colorectal cancer, chemoresistance

\section{Introduction}

Colorectal cancer (CRC) is one of the most commonly diagnosed cancer types, and is a leading cause of cancer-related mortality. Although surgical resection is an optimal therapeutic strategy during the early stages of CRC, chemotherapy remains an important treatment option for patients diagnosed at an advanced stage. Indeed, platinum-based chemotherapy, including cisplatin (DDP), is usually adopted for treating CRC (1). However, a large proportion of patients with cancer eventually relapse and develop drug resistance, despite an initial response to DDP (2). Recently, the co-administration of DDP along with other anticancer agents has been proven to be a more effective treatment strategy $(3,4)$. Therefore, understanding the potential mechanisms underlying the development of DDP resistance may aid in the design of combined chemotherapy regimens.

Chemotherapy usually induces tumor microenvironment remodeling to sustain the cellular hierarchy of the tumor via secreting cytokines. The tumor microenvironment is characterized by proinflammatory mediator accumulation and cell infiltration. A significant correlation has been demonstrated between chronic inflammation and tumor invasion, metastasis and chemoresistance (5). Interleukin (IL)-17 is a proinflammatory cytokine that is secreted by T-helper 17 (Th17) cells and serves an important role in the host defense in inflammatory conditions and in cancer development, including inflammatory bowel disease (6), rheumatoid arthritis (7), lung cancer (8) and thyroid cancer (9). Indeed, interleukin-17 (IL-17) has a pleiotropic function in tumor initiation, development and metastasis (10-15). Notably, IL-17 has been suggested to serve a crucial role in promoting chemoresistance in tumor cells. In breast cancer, tumor-infiltrating $\mathrm{T}$ lymphocytes were shown to produce significant amounts of IL-17, further upregulating phosphorylated extracellular signal-regulated protein kinases 1 and 2 (ERK1/2) and activating the mitogen-activated protein kinase (MAPK) pathway. Furthermore, IL-17 was found to be able to promote the migration and invasion of breast cancer cells (16).

Similarly, IL-17 is key to the initiation and development of CRC. Exogenous IL-17 may facilitate the self-renewal and invasion of cancer-initiating cells. However, the role of IL-17 in DDP resistance in CRC has not been fully elucidated. The present study evaluated IL-17 production among clinical CRC 
samples and its association with chemoresistance. Furthermore, the CRC HCT116 line was used to investigate the biological function of IL-17 in facilitating the development of DDP resistance in CRC.

\section{Materials and methods}

Specimens. A total of 37 pairs of colon tumor tissue samples and corresponding para-carcinoma tissue samples were collected from 37 patients (mean age, 55.82 \pm 13.61 years; age range, 49-71 years) during radical resection of colorectal cancer in Weihai Central Hospital (Weihai, Shandong, China) between November 2015 and December 2016. All the patients in this cohort were pathologically diagnosed with colon cancer, and had not received any preoperative radiotherapy and/or chemotherapy. Detailed clinical characteristics and pathological parameters were collected, and all the patients provided written informed consent for their data to be used in the present study. The study was approved by the Ethical Committee of Weihai Central Hospital.

Tissue sections and immunohistochemical staining. Formalin-fixed (10\%) paraffin-embedded tissue blocks (fixed at room temperature for $24 \mathrm{~h}$ ) were cut into $3-\mu \mathrm{m}$ sections. Following dewaxing in xylene and hydration through graded ethanol, the sections were immersed in $3 \% \mathrm{H}_{2} \mathrm{O}_{2}$ at room temperature for $15 \mathrm{~min}$ to block endogenous peroxidase binding, followed by boiling in ethylenediaminetetraacetic acid-alkaline solution for antigen retrieval. The sections were then blocked at room temperature for $10 \mathrm{~min}$ using 5\% animal normal serum (cat. no. C0265; Beyotime Institute of Biotechnology, Shanghai, China). A monoclonal rabbit anti-human IL-17 primary antibody (cat. no. ab79056; 1:50; Abcam, Cambridge, MA, USA) was incubated with the slides overnight at $4^{\circ} \mathrm{C}$. The following day, the sections were washed and incubated with horse peroxidase (HRP)-conjugated goat anti-rabbit secondary antibodies (cat. no. ab6721; 1:100; Abcam) at room temperature for $30 \mathrm{~min}$. The slides were then rinsed with $0.05 \%$ Tween-phosphate-buffered saline (PBS), and treated with diluted streptavidin-horseradish peroxidase for $30 \mathrm{~min}$ at room temperature. The slides were washed with $0.05 \%$ Tween-PBS buffer. Finally, the sections were stained with diaminobenzidine (DAB) for $90 \mathrm{sec}$ at room temperature, and stained with hematoxylin for $60 \mathrm{sec}$ at room temperature and fixed in $60 \%$ neutral balata for $12 \mathrm{~h}$. The expression of IL-17 in the tissue samples was determined by the intensity score of the IHC image: A score of 0 was defined as negative expression, and 1,2 and 3 as weak expression, moderate expression and strong expression, respectively, by visually analyzing the intensity of the staining. Five randomly selected fields of each sample were picked, and the sum of the intensity scores was calculated. A total score of between 0 and 2 was defined as negative expression, 3 and 4 were defined as weak expression, and a score of $>5$ was defined as strong expression.

Cell culture. The human CRC HCT116 cell line (purchased from Chinese Academy of Sciences Cell Bank, Shanghai, China) was cultured with RPMI-1640 (Invitrogen; Thermo Fisher Scientific, Inc., Waltham, MA, USA) plus $10 \%$ fetal bovine serum (Invitrogen; Thermo Fisher Scientific, Inc.) in a humidified incubator at $37^{\circ} \mathrm{C}\left(5 \% \mathrm{CO}_{2}\right)$. To examine the inhibitory effects of DDP on HCT116 cells, cells were seeded on 96 well plates with the density of $5 \times 10^{4}$ and treated with RPMI-1640 containing 100, 200, 400 or $800 \mathrm{nM}$ DDP. To determine the effect of IL-17 on HCT116 cells, cells were seeded on 12 well plates with the density of $5 \times 10^{5}$, and the treatments included DDP alone $(400 \mathrm{nM})$, or a combination of DDP and IL-17 (50 ng/ml) or IL-17 inhibitors (IL-17a and IL-17 monoclonal antibody Secukinumab; cat. no. NDC:0078-0639; Novartis International AG, Basel, Switzerland; 4 mg/ml).

Assay for cell viability. The MTT assay (Invitrogen; Thermo Fisher Scientific, Inc.) was used to monitor the cell viability of each group. Briefly, HCT116 cells were first washed with buffer (PBS; pH 7.4), trypsinized, washed, counted and then re-seeded into a 96-well plate. Next, $10 \mu \mathrm{l}$ MTT reagent was added and the plate was cultured at $37^{\circ} \mathrm{C}$ in a humidified incubator $\left(5 \% \mathrm{CO}_{2}\right)$ until a purple precipitate appeared. Thereafter, $100 \mu$ l detergent solution was added and the plate was incubated for $2 \mathrm{~h}$ in the dark. Absorbance was detected at $570 \mathrm{~nm}$ using a microplate reader.

Western blot analysis. Treated cells were washed in PBS, immediately followed by cell use of extraction buffer (Thermo Fisher Scientific, Inc.) with a protease inhibitor cocktail (Roche Diagnostics, Basel, Switzerland) for cell lysis, according to the manufacturer's protocol. The quantification of protein was further determined using a Bradford protein assay kit (Bio-Rad Laboratories, Inc., Hercules, CA, USA). Proteins (20 $\mu \mathrm{g}$ for each sample) were separated by $10 \%$ SDS-PAGE and then transferred to a polyvinylidene difluoride membrane, which was blocked by $5 \%$ skimmed milk (suspended in TBS with $0.05 \%$ Tween-20) at room temperature for $1 \mathrm{~h}$, and then incubated with the primary antibodies against phosphorylated protein kinase B (p-Akt; cat. no. ab38449; 1:1,000 dilution), apoptosis regulator BAX (Bax; cat. no. ab32503; 1:1,000 dilution), apoptosis regulator Bcl-2 (Bcl-2; cat. no. ab59348; 1:1,000 dilution) and serine/threonine-protein kinase mTOR (mTOR; cat. no. ab2732; 1:2,000 dilution; all Abcam) proteins at $4^{\circ} \mathrm{C}$ overnight. $\beta$-actin (cat. no. ab8227; 1:1,000; Abcam) served as the internal control. The following day, the membrane was washed and chemiluminescence was measured following incubation with the goat anti-rabbit HRP-conjugated (IgG H\&L) secondary antibodies (cat. no. ab6721; 1:5,000; Abcam). The membranes were then incubated with Enhanced Chemiluminescence Detection kit (Vazyme Biotech Co., Ltd., Nanjing, China) The relative expression of p-Akt, Bax, Bcl-2 and mTOR proteins to the expression of $\beta$-actin was statistically evaluated by ImageJ (version 1.45f; National Institutes of Health, Bethesda, MD, USA).

Cell apoptosis analysis. Once the cells had been treated with DPP with or without IL-17 for $72 \mathrm{~h}$, flow cytometry with the Annexin V-fluorescein isothiocyanate (FITC)/propidium iodide (PI) staining method was employed to monitor cell apoptosis in each group. Briefly, the cells were washed, trypsinized and resuspended in the staining solution provided with the Annexin V-FITC Apoptosis Detection kit (Invitrogen; Thermo Fisher Scientific, Inc.), according to the manufacturer's 

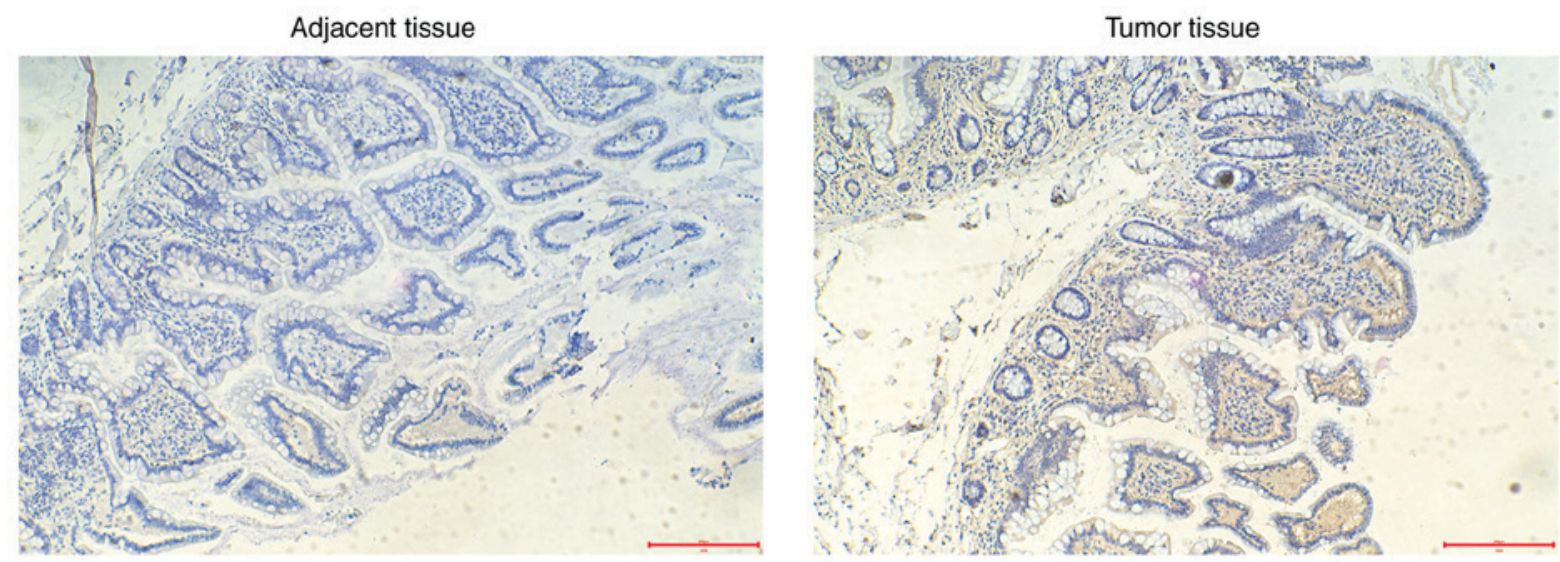

Figure 1. Relative expression of interleukin-17 in colorectal cancer tumor tissue and adjacent tissue. Scale bar, $200 \mu \mathrm{M}$.

protocol. Subsequent to incubation for $1 \mathrm{~h}$, cell apoptosis was measured by a flow cytometer (BD Biosciences, Franklin Lakes, NJ, USA). Cells with a positive Annexin V-FITC signal and a negative PI signal were considered to be apoptotic.

Statistical analysis. All experiments were verified with repetition in triplicate. Final data were statistically analyzed using SPSS 22.0 software (IBM Corp., Armonk, NY, USA). All data are presented as the mean \pm standard deviation. Differences among multiple groups were analyzed by one-way analysis of variance followed by Dunnett's post-hoc test. Results with a P-value of $<0.05$ were considered to be statistically significant.

\section{Results}

Association of IL-17 with clinical and pathological parameters in patients with $C R C$. To determine IL-17 expression in CRC tissue, CRC samples from 37 patients collected during surgery were included in the present study. Immunohistochemical staining was used to identify the positivity of IL-17 (Fig. 1). The clinical and pathological characteristics, and the IL-17 expression data, are summarized in Table I. The expression levels of IL-17, stratified by sex, age, tumor size, tumor differentiation, invasion depth, lymph node metastasis and distant metastasis, were determined. Notably, IL-17 expression was positively associated with the tumor size $(\mathrm{P}=0.011)$, poor tumor cell differentiation $(\mathrm{P}=0.025)$, a greater depth of invasion $(\mathrm{T} 3-\mathrm{T} 4 ; \mathrm{P}=0.007)$ and the presence of lymph node metastasis $(\mathrm{N} 1-\mathrm{N} 3 ; \mathrm{P}=0.034)$. These results indicated a positive association between IL-17 expression and CRC development.

DDP inhibits the viability of $C R C$ cells. DDP was reported to exert a potent inhibitory effect against the growth of CRC. To further determine the optimal half maximal inhibitory concentration of DDP for HCT116 cells, a panel of serial 2-fold diluted concentration (100-800 nM) of DDP was added to the cell culture for $72 \mathrm{~h}$. The MTT assay was used to evaluate cell viability at $0,6,12,24,48$ and 72 h. As shown in Fig. 2, $100 \mathrm{nM}$ DDP was sufficient to inhibit $>20 \%$ of cell proliferation by $72 \mathrm{~h}$, while $800 \mathrm{nM}$ DDP successfully inhibited $60 \%$ of cell proliferation by $72 \mathrm{~h}$. Based on these results, $400 \mathrm{nM}$ inhibited $\sim 50 \%$ of cell proliferation and it was selected as the optimal concentration to further determine the role of IL-17 in the DDP resistance of CRC cells.

IL-17 facilitates DDP resistance in CRC cells. To determine whether IL-17 facilitated the development of CRC resistance to DDP, HCT116 cells were treated with DDP, DDP plus IL-17 or DDP plus IL-17a. An additional group of HCT116 cells without any treatment was used as a negative control. At $72 \mathrm{~h}$ post-treatment, compared with the negative control group, the DDP-treatment group exhibited 53.6 $\pm 8.6 \%$ inhibition of proliferation. The addition of IL-17 was able to reduce inhibition of cellular proliferation to $26.9 \pm 4.29 \%$, while IL-17a treatment further promoted the inhibition of cell proliferation to $76.35 \pm 11.43 \%$ (Fig. 3A and B).

IL-17 inhibits the apoptosis of CRC cells. Given the observation that IL-17 was able to inhibit the proliferation of HCT116 cells, whether IL-17 could inhibit the apoptosis of CRC cells was further determined. Using Annexin V-FITC/PI staining, the percentage of apoptotic HCT116 cells was determined. As shown in Fig. $4 \mathrm{~A}$ and $\mathrm{B}$, the apoptosis rate in the negative control group (HCT166 cells only) was $2.03 \pm 1.03 \%$. DDP treatment increased the apoptosis rate to $40.16 \pm 6.26 \%$, while adding IL-17 reduced the apoptosis rate to $20.95 \pm 3.49 \%$ and inhibiting IL-17 signaling in DDP-treated HCT116 cells increased the apoptosis rate to $68.33 \pm 8.68 \%$. These results suggested that IL-17 signaling facilitated DDP resistance in CRC cells, while inhibiting IL-17 signaling via treatment with an anti-IL-17 antibody was able to markedly improve the efficacy of DDP.

$I L-17$ promotes the expression of $p-A k t, B c l-2$ and mTOR, and inhibits Bax expression in CRC cells. To investigate the molecular mechanisms underlying the inhibitory effect of IL-17 in terms of the DDP resistance of CRC cells, western blot analysis was used to determine the expression of key apoptosis-related proteins (Fig. 5A and B). As shown in Fig. 5A and B, Bax was significantly downregulated, and p-Akt, mTOR and Bcl-2 were significantly upregulated in the tumor tissues compared with the adjacent normal tissues. Moreover, compared with the negative control group, DDP treatment induced a significant reduction in the expression of p-Akt, Bcl-2 and mTOR, along with significantly increased 
Table I. Association of IL-17 expression with clinical and pathological parameters in patients with colorectal cancer.

\begin{tabular}{|c|c|c|c|c|c|}
\hline \multirow[b]{2}{*}{ Factors } & \multirow[b]{2}{*}{ Cases, $\mathrm{n}$} & \multicolumn{3}{|c|}{ IL-17 expression, $\mathrm{n}$} & \multirow[b]{2}{*}{ P-value } \\
\hline & & Negative & Weak & Strong & \\
\hline Total patients & & 11 & 14 & 12 & \\
\hline Sex & & & & & 0.818 \\
\hline Male & 23 & 6 & 9 & 8 & \\
\hline Female & 14 & 5 & 5 & 4 & \\
\hline Age, years & & & & & 0.177 \\
\hline$<60$ & 25 & 8 & 7 & 10 & \\
\hline$\geq 60$ & 12 & 3 & 7 & 2 & \\
\hline Tumor size, $\mathrm{cm}^{3}$ & & & & & 0.011 \\
\hline$\geq 5$ & 15 & 2 & 4 & 9 & \\
\hline$<5$ & 22 & 9 & 10 & 3 & \\
\hline Tumor differentiation & & & & & 0.025 \\
\hline Well, moderate & 16 & 8 & 6 & 2 & \\
\hline Poor & 21 & 3 & 8 & 10 & \\
\hline Invasion depth & & & & & 0.007 \\
\hline $\mathrm{T} 1-\mathrm{T} 2$ & 19 & 6 & 11 & 2 & \\
\hline T3-T4 & 18 & 5 & 3 & 10 & \\
\hline Lymph node metastasis & & & & & 0.034 \\
\hline N0 & 15 & 8 & 4 & 3 & \\
\hline N1-N3 & 22 & 3 & 10 & 9 & \\
\hline Distant metastasis & & & & & 0.332 \\
\hline No & 17 & 7 & 6 & 4 & \\
\hline Yes & 20 & 4 & 8 & 8 & \\
\hline
\end{tabular}

IL-17, interleukin-17.

Bax expression (Fig. 5C and D). The addition of IL-17 to the DPP-treated cells was able to restore the expression of p-Akt, Bcl-2 and mTOR, and to inhibit Bax expression. Inhibition of IL-17 signaling (DDP + IL-17a) in the HCT116 cells caused further suppression of the expression of p-Akt, Bcl-2 and mTOR, and promoted the expression of Bax, compared with that in the group treated with DDP and IL-17 (Fig. 5C and D). These results indicated the mechanism that underlies IL-17 promoting the development of DDP resistance.

\section{Discussion}

As CRC is one of the most prevalent and lethal cancer types worldwide, its treatment is challenging. DDP is a potent anticancer agent and has been widely applied in the treatment of CRC in clinical practice (17). However, DDP resistance is one of the most challenging problems encountered during CRC treatment. The present study observed elevated IL-17 expression in tumor tissues collected from patients with CRC. Furthermore, the findings indicated that IL-17 may be one of the key factors mediating DDP resistance, and inhibition of IL-17 signaling in CRC cell culture was able to reverse resistance to DDP, at the biological functional and molecular levels. First, the addition of IL-17 to DPP-treated cells was able

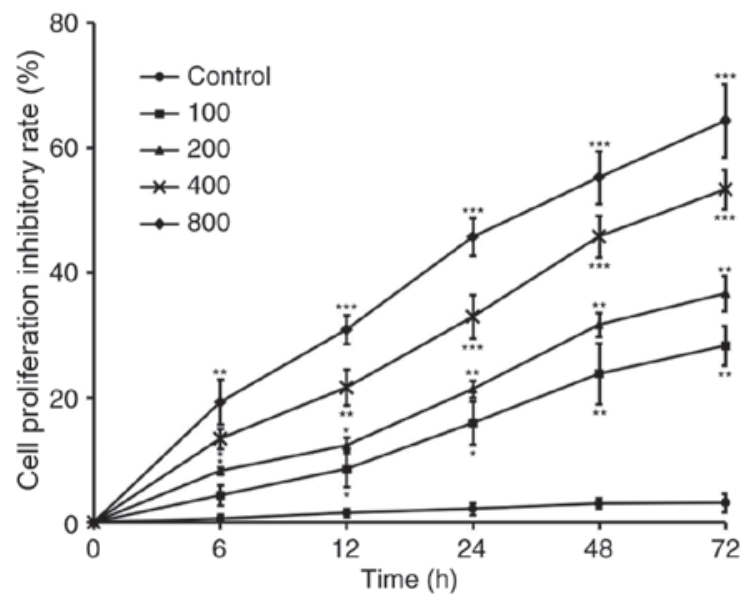

Figure 2. DDP inhibits the proliferation of colorectal cancer cells. To determine the optimal half maximal inhibitory concentration of DDP, serially diluted DDP (0-800 nM, 2-fold diluted) was used to treat HCT116 cells, and the inhibitory percentage of cell proliferation was measured at different time points $\left(0,6,12,24,48\right.$ and 72 h). ${ }^{*} \mathrm{P}<0.05,{ }^{* *} \mathrm{P}<0.01$ and ${ }^{* * * *} \mathrm{P}<0.001$ vs. control. DDP, cisplatin.

to inhibit apoptosis and promote HCT116 cell growth, while inhibition of IL-17 signaling exerted a synergistic inhibitory effect on CRC cell proliferation. 


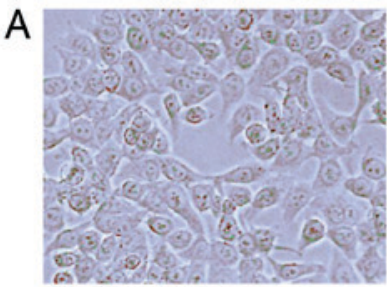

Control

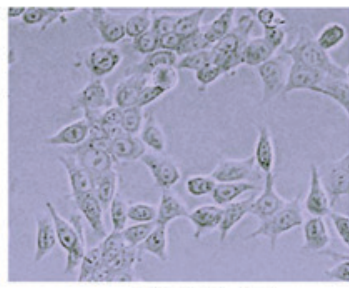

DDP+IL-17

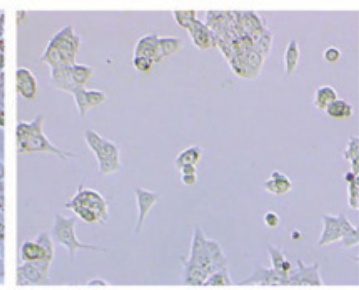

DDP

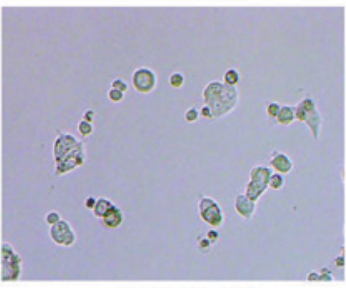

DDP+IL- $17 \alpha$
B

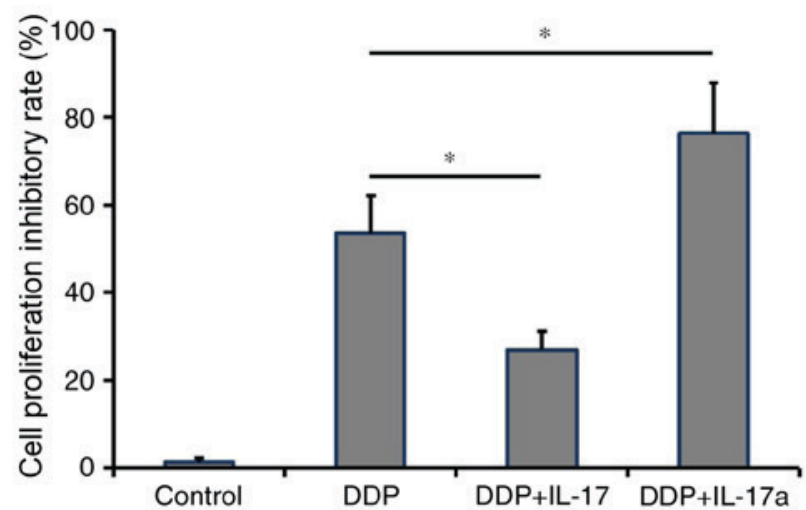

Figure 3. IL-17 facilitates DDP resistance in colorectal cancer cells. (A) Proliferation of HCT116 cells following DDP, DDP plus IL-17 and DDP plus IL-17a treatment for $72 \mathrm{~h}$. (B) Quantification of inhibitory percentage of cell proliferation following DDP, DDP plus IL-17 and DDP plus IL-17a treatment for $72 \mathrm{~h}$. ${ }^{*} \mathrm{P}<0.05$ vs. control. DDP, cisplatin; IL-17, interleukin 17; IL-17a, interleukin 17 antibody.
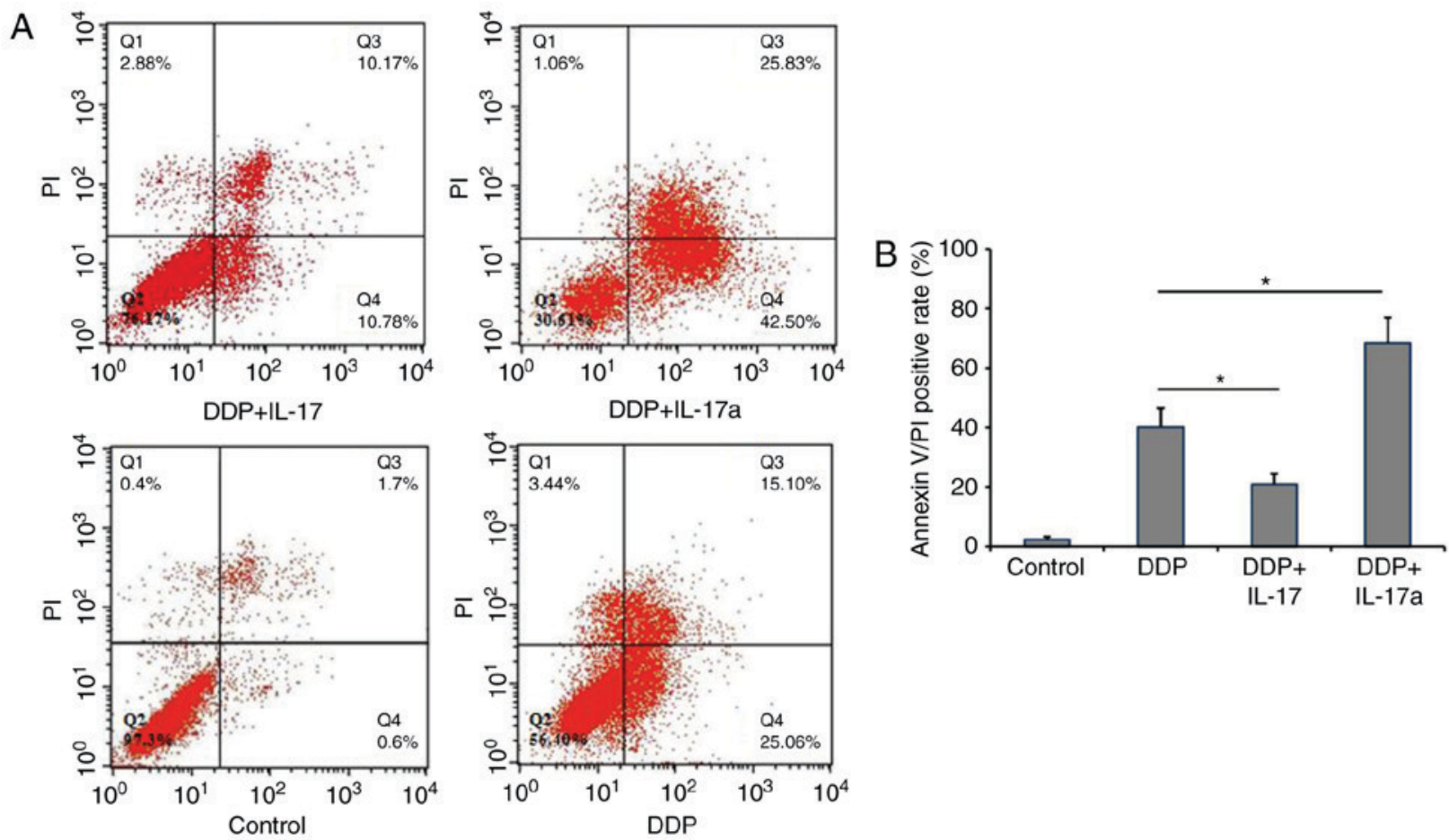

Figure 4. IL-17 inhibits the apoptosis of colorectal cancer cells. (A) Flow cytometry analysis determined the apoptosis of HCT116 cells treated with DDP, DDP plus IL-17 and DDP plus IL-17a. HCT116 cells without treatment were used as a negative control. (B) Quantification of HCT116 cell apoptosis following DDP, DDP plus IL-17 and DDP plus IL-17a treatment, as demonstrated by the Annexin V/PI assay. "P<0.05. DDP, cisplatin; IL-17, interleukin 17; IL-17a, interleukin 17 antibody; PI, propidium iodide.

The molecular mechanism through which IL-17 promotes the development of DDP resistance was also investigated. It was found that IL-17 promotes cell proliferation and inhibits apoptosis by enhancing the expression of p-Akt, mTOR and $\mathrm{Bcl}-2$, and suppressing the expression of Bax. The phosphoinositide 3-kinase (PI3K)-Akt-mTOR pathway is often found to be constitutively activated in human tumor cells, integrating environmental and intracellular signals to support cell growth (18). Also referred to as a Bcl-2-like protein, Bax is a key pro-apoptotic member of the $\mathrm{Bcl}-2$ protein family.
$\mathrm{Bcl}-2$ is localized in the outer mitochondrial membrane, where it serves a key role in promoting cell survival and inhibiting the function of pro-apoptotic proteins (19). These findings were also consistent with those of the present study, where IL-17 was revealed to enhance the proliferation and inhibit the apoptosis of DPP-treated HCT166 cells. The present findings further confirmed that the inhibition of IL-17 is a promising therapeutic target for DDP resistance in CRC.

The role of IL-17 in promoting chemoresistance and cancer cell proliferation was also investigated in other types 

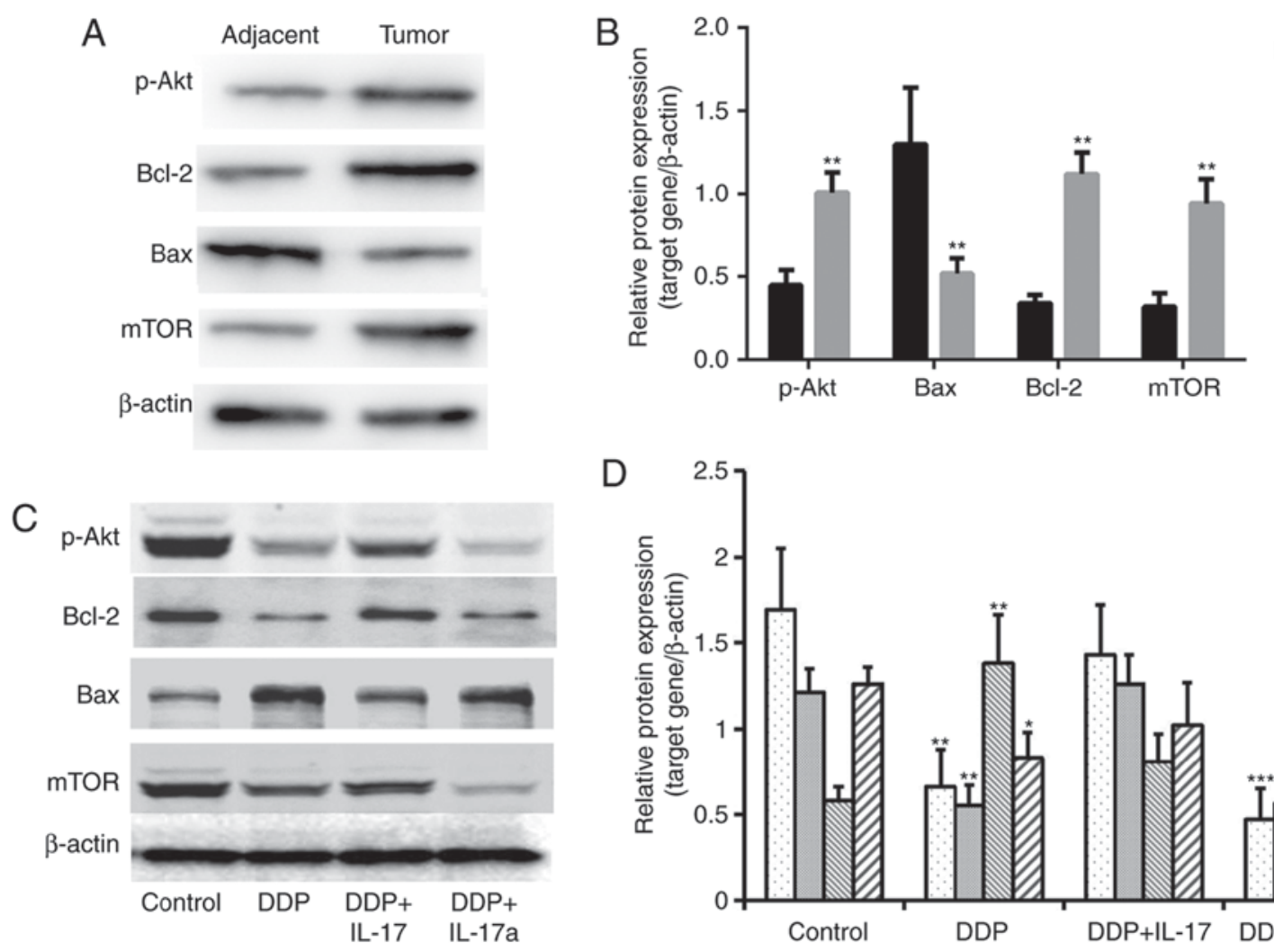

Adjacent

Tumor
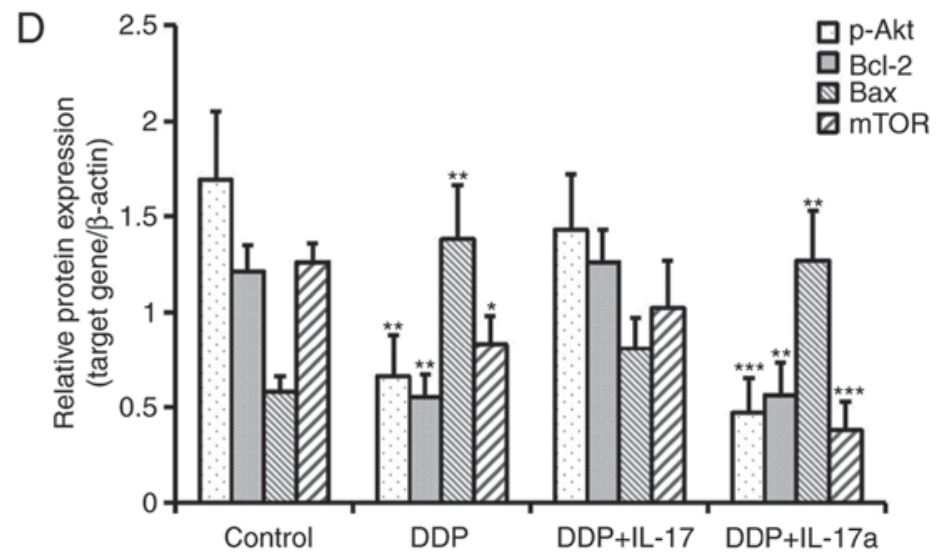

Figure 5. IL-17 promotes the expression of p-Akt, Bax and mTOR in colorectal cancer cells. (A) Western blot analysis revealed elevated levels of p-Akt, Bcl-2 and mTOR, and decreased levels of Bax in tumor tissues compared with that in the adjacent tissues. (B) Semi-quantification of each protein expression level in the different groups tested in panel (A). (C) Western blot analysis revealed elevated levels of p-Akt, Bcl-2 and mTOR following the addition of IL-17 to HCT116 cells treated with DDP. (D) Semi-quantification of each protein expression level in the different groups tested in panel (A). $\mathrm{P}<0.05$, ${ }^{* *} \mathrm{P}<0.01$ and ${ }^{* * *} \mathrm{P}<0.001$ vs. control. DDP, cisplatin; IL-17, interleukin 17; IL-17a, interleukin 17 antibody; p-Akt, phosphorylated protein kinase B; Bcl-2, apoptosis regulator Bcl-2; Bax, apoptosis regulator BAX; mTOR, serine/threonine-protein kinase mTOR.

of cancer. In breast cancer, IL-17 levels and IL-17-secreting cells were found to be increased in tumor tissues and were correlated with a poor prognosis (20). Recombinant IL-17 was shown to trigger the MAPK pathway by activation of ERK1/2 signaling in breast cancer, therefore promoting cancer cell proliferation and chemotherapy resistance (16). In non-small-cell lung cancer, IL-17 was able to promote lung cancer growth by promoting inflammation, which contributes to resistance to programmed cell death protein 1 blockade, and tumor sensitivity to cytokine depletion (8). The present study identified a novel mechanism mediated by IL-17 to promote DDP resistance via promoting PI3K-Akt-mTOR signaling and suppressing Bcl-2 expression, which may assist in the design of novel therapeutic strategies to overcome DDP resistance.

A notable observation from the clinical samples was that elevated IL-17 was present in CRC tissues, consistent with previous findings. Lereclus et al (21) suggested that the baseline level of serum IL-17 concentration is an important factor that is significantly associated with the response of patients with metastatic CRC to bevacizumab, a monoclonal antibody that targets vascular endothelial growth factor (21). However, the mechanism underlying the elevated IL-17 levels observed in the tumor remains unclear. Al-Samadi et al (22) reported that the numbers of Th17 cells secreting IL-17 were increased in the stroma, as well as in the adenomatous/cancerous epithelium (22). However, additional studies are required to elucidate the mechanism underlying the significantly increased numbers of Th17 cells and IL-17 levels present in CRC.

In conclusion, the present study identified an important role for IL-17 in the DDP resistance of CRC. The addition of IL-17 inhibited the apoptosis and promoted the proliferation of a CRC cell line treated with DDP, while the inhibition of IL-17 signaling alongside DDP treatment synergistically increased cell apoptosis. Finally, IL-17 may facilitate chemotherapy resistance via promoting PI3K-Akt-mTOR and suppressing Bcl-2 signaling. The findings of the present study provide solid experimental evidence for the design of novel combined chemotherapy regimens.

\section{Acknowledgements}

Not applicable.

\section{Funding}

No funding was received.

\section{Availability of data and materials}

The datasets used and/or analyzed during the current study are available from the corresponding author on reasonable request. 


\section{Authors' contributions}

BZ designed the experiments, and wrote and revised the paper. GS conducted the majority of the experiments, analyzed the data and also wrote a portion of the manuscript. YQ, HY and QK conducted a number of the experiments and analyzed a portion of the data.

\section{Ethics approval and consent to participate}

All the patients provided written informed consent for their data to be used in the present study. The study was approved by the Ethical Committee of Weihai Central Hospital.

\section{Patient consent for publication}

Informed consent was obtained from each patient.

\section{Competing interests}

The authors declare that they have no competing interests.

\section{References}

1. McQuade RM, Stojanovska V, Bornstein JC and Nurgali K Colorectal cancer chemotherapy: The evolution of treatment and new approaches. Curr Med Chem 24: 1537-1557, 2017.

2. Galluzzi L, Vitale I, Michels J, Brenner C, Szabadkai G, Harel-Bellan A, Castedo M and Kroemer G: Systems biology of cisplatin resistance: Past, present and future. Cell Death Dise 5: e1257, 2014.

3. Valsecchi ME: Combined nivolumab and ipilimumab or monotherapy in untreated melanoma. N Eng J Med 373: 1270, 2015.

4. Wu YL, Zhou C, Hu CP, Feng J, Lu S, Huang Y, Li W, Hou M, Shi JH, Lee KY, et al: Afatinib versus cisplatin plus gemcitabine for first-line treatment of Asian patients with advanced non-small-cell lung cancer harbouring EGFR mutations (LUX-Lung 6): An open-label, randomised phase 3 trial. Lancet Oncol 15: 213-222, 2014.

5. Landskron G, De la Fuente M, Thuwajit P, Thuwajit C and Hermoso MA: Chronic inflammation and cytokines in the tumor microenvironment. J Immunol Res 2014: 149185, 2014.

6. Fujino S, Andoh A, Bamba S, Ogawa A, Hata K, Araki Y, Bamba T and Fujiyama Y: Increased expression of interleukin 17 in inflammatory bowel disease. Gut 52: 65-70, 2003.

7. Chabaud M, Garnero P, Dayer JM, Guerne PA, Fossiez F and Miossec P: Contribution of interleukin 17 to synovium matrix destruction in rheumatoid arthritis. Cytokine 12: 1092-1099, 2000.

8. Akbay EA, Koyama S, Liu Y, Dries R, Bufe LE, Silkes M, Alam MM, Magee DM, Jones R, Jinushi M, et al: Interleukin-17A Promotes lung tumor progression through neutrophil attraction to tumor sites and mediating resistance to PD-1 blockade. J Thorac Oncol 12: 1268-1279, 2017
9. Carvalho DFG, Zanetti BR, Miranda L, Hassumi-Fukasawa MK, Miranda-Camargo F, Crispim JCO and Soares EG: High IL-17 expression is associated with an unfavorable prognosis in thyroid cancer. Oncol Lett 13: 1925-1931, 2017.

10. Wilke CM, Kryczek I, Wei S, Zhao E, Wu K, Wang G and Zou W: Th17 cells in cancer: Help or hindrance? Carcinogenesis 32: 643-649, 2011

11. Hu J, Ye H, Zhang D, Liu W, Li M, Mao Y and Lu Y: U87MG glioma cells overexpressing IL-17 acclerate early-stage growth in vivo and cause a higher level of CD31 mRNA expression in tumor tissues. Oncol Lett 6: 993-999, 2013.

12. Ernst M and Putoczki T: IL-17 cuts to the chase in colon cancer. Immunity 41: 880-882, 2014.

13. Wang K, Kim MK, Di Caro G, Wong J, Shalapour S, Wan J, Zhang W,Zhong Z,Sanchez-Lopez E, Wu LW, et al: Interleukin-17 receptor a signaling in transformed enterocytes promotes early colorectal tumorigenesis. Immunity 41: 1052-1063, 2014.

14. Chang Y, Al-Alwan L, Risse PA, Halayko AJ, Martin JG, Baglole CJ, Eidelman DH and Hamid Q: Th17-associated cytokines promote human airway smooth muscle cell proliferation. FASEB J 26: 5152-5160, 2012.

15. Yang B, Kang H, Fung A, Zhao H, Wang T and Ma D: The role of interleukin 17 in tumour proliferation, angiogenesis, and metastasis. Mediators Inflamm 2014: 623759, 2014.

16. Cochaud S, Giustiniani J, Thomas C, Laprevotte E, Garbar C, Savoye AM, Curé H, Mascaux C, Alberici G, Bonnefoy N, et al: IL-17A is produced by breast cancer TILs and promotes chemoresistance and proliferation through ERK1/2. Sci Rep 3: 3456, 2013.

17. Boulikas T and Vougiouka M: Cisplatin and platinum drugs at the molecular level. (Review). Oncol Rep 10: 1663-1682, 2003.

18. Zhang HB, Lu P, Guo QY, Zhang ZH and Meng XY: Baicalein induces apoptosis in esophageal squamous cell carcinoma cells through modulation of the PI3K/Akt pathway. Oncol Lett 5: 722-728, 2013.

19. Al-Fatlawi AA, Al-Fatlawi AA, Irshad M,Zafaryab M, Rizvi MM and Ahmad A: Rice bran phytic acid induced apoptosis through regulation of $\mathrm{Bcl}-2 / \mathrm{Bax}$ and 53 genes in $\mathrm{HepG} 2$ human hepatocellular carcinoma cells. Asian Pac J Cancer Prev 15: 3731-3736, 2014.

20. Ji Y and Zhang W: Th17 cells: Positive or negative role in tumor? Cancer Immunol Immunother 59: 979-987, 2010.

21. Lereclus E, Tout M, Girault A, Baroukh N, Caulet M, Borg C, Bouché O, Ternant D, Paintaud G, Lecomte T and Raoul W: A possible association of baseline serum IL-17A concentrations with progression-free survival of metastatic colorectal cancer patients treated with a bevacizumab-based regimen. BMC Cancer 17: 220, 2017.

22. Al-Samadi A, Moossavi S, Salem A, Sotoudeh M, Tuovinen SM, Konttinen YT, Salo T and Bishehsari F: Distinctive expression pattern of interleukin-17 cytokine family members in colorectal cancer. Tumor Bio 37: 1609-1615, 2016.

(i)(3) This work is licensed under a Creative Commons Attribution-NonCommercial-NoDerivatives 4.0 International (CC BY-NC-ND 4.0) License. 